\section{Necrolisis epidérmica tóxica en el curso de un síndrome hemofagocítico secundario a linfoma de Hodgkin. Caso clínico}

\author{
CAMILA PEÑA ${ }^{1}$, JAVIER VOISIN $^{a}$, \\ JOSÉ TOMÁS GONZÁLEZ ${ }^{\mathrm{a}}$, PABLO VILLEGAS ${ }^{2}$
}

\section{Toxic epidermal necrolysis during a hemophagocytic syndrome secondary to Hodgkin lymphoma. Report of one case}

Toxic epidermal necrolysis (TEN) is a lethal entity, characterized by extensive epidermal necrosis and multiorgan failure. Hemophagocytic syndrome (HFS) is also a rare and lethal syndrome characterized by hyperinflammation that leads to the appearance offever, pancytopenia, organomegaly and hemophagocytosis. The concomitance of these diseases is extremely uncommon. We report a 38 years old female, who during the course of a HFS secondary to Hodgkin Lymphoma (HL), presented a TEN secondary to antibiotics. She was admitted due to a consumptive syndrome, lymphadenopathy, visceromegaly and severe pancytopenia. Laboratory and bone marrow tests confirmed HFS. Due to constant fever, imipenem was indicated. On the third day she started with pain and skin rash. She evolved with positive Nikolsky sign. Cutaneous biopsy was concordant with extensive TEN, which was managed with intravenous immunoglobulin and dexamethasone. A complete response and normalization of the blood count were achieved. Finally, the lymph node biopsy showed HL of mixed cellularity type, which was managed with 8 cycles of ABVD chemotherapy, achieving complete remission.

(Rev Med Chile 2018; 146: 523-527)

Key words: Hodgkin Disease; Lymphohistiocytosis, Hemophagocytic; Stevesns-Johnson Syndrome.

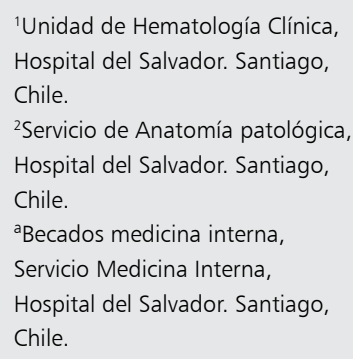

Sin apoyo financiero.

Autores declaran no tener conflictos de interés.

Recibido el 14 de diciembre de 2017, aceptado el 2 de mayo de 2018.

\section{Correspondencia a: \\ Dra. Camila Peña O. \\ camipena@gmail.com}

\section{L}

a necrolisis epidérmica tóxica (NET) es una entidad infrecuente y letal, caracterizada por $\checkmark$ necrosis extensa de la epidermis y falla multiorgánica. Por otro lado, el síndrome hemofagocítico (SHF) es también una rara y grave patología. Este se caracteriza por hiperinflamación, fiebre, pancitopenia, visceromegalia y hemofagocitosis. Una de las causas más frecuentes de este último son las neoplasias hematológicas, siendo el linfoma de Hodgkin (LH) una causa poco común.

La concomitancia de ambos síndromes es extremadamente infrecuente.

Presentamos el caso de una paciente joven, que durante el curso de un SHF secundario a LH presentó un NET secundario a antibióticos.

\section{Caso clínico}

Mujer de 38 años. Ingresó por fiebre, síndrome consuntivo de 2 meses de evolución y pancitopenia. Al ingreso destacó al examen físico: ictericia, adenopatía cervical izquierda de $1,5 \mathrm{~cm}$ y supraclavicular izquierda de $2 \mathrm{~cm}$. Hígado a $3 \mathrm{~cm}$ bajo reborde costal (BRC). En el scanner (TAC) se evidenciaron múltiples adenopatías en mediastino de hasta $2,2 \mathrm{~cm}$, axilares $3,2 \mathrm{~cm}$ y retroperitoneales 
múltiples de hasta $2 \mathrm{~cm}$. Esplenomegalia $17 \mathrm{~cm}$ y hepatomegalia. Dentro de los exámenes destacó $\mathrm{Hb} 9,1 \mathrm{~g} / \mathrm{L}$, recuento de neutrófilos de $427 \mathrm{~mm}^{3}$ y de plaquetas $32.000 \mathrm{~mm}^{3}$. Además Bilirrubina Total de 2,8 md/dL, Fosfatasa Alcalina de 728 UI/L, LDH de 665 UI/L, ferritina de $1692 \mathrm{ng} /$ $\mathrm{mL}$. Con esto se decidió practicar mielograma y biopsia de médula ósea (MO) para evaluar la pancitopenia, suponiendo un diagnóstico de linfoma con infiltración de MO e hígado. Sin embargo, se encontró una MO con alta celularidad, displasia en las tres series y hemofagocitosis. Dada la alta sospecha en ese momento se realizó extensión de los exámenes destacando triglicéridos (TG) de $505 \mathrm{mg} / \mathrm{dL}$ y fibrinógeno (Fg) de $256 \mathrm{mg} / \mathrm{dL}$. Se hizo diagnóstico de SHF, ya que cumplió 6 criterios. El estudio reumatológico fue negativo. Los virus VIH, VHB y VHC resultaron también negativos. Quantiferón negativo, hemomac nega- tivo y CMV negativa. En espera de la biopsia de alguna adenopatía, se mantuvo febril, requiriendo varios esquemas antibióticos. Luego del inicio de Imipenem evolucionó con dolor generalizado, exantema y signo Nikolsky positivo. Se sospechó NET, que fue corroborado luego también por biopsia cutánea. En 2 días evolucionó con extensa necrosis de epidermis (Figura 1) sin afectación mucosa, y fue manejada en la unidad de paciente crítico. Se administró inmunoglobulina intravenosa (IgIV) y dexametasona endovenosa, con excelente respuesta. Se recuperó de su cuadro cutáneo y el hemograma se normalizó, lo que nos dio un puente para realizar la biopsia de ganglio, que finalmente mostró Linfoma de Hodgkin (LH) clásico tipo celularidad mixta. Estudio de VEB en la biopsia dio positivo para EBER (del inglés EBV-encoded small nuclear RNA) por CISH (Figura 2). Se realizó posteriormente EBER por

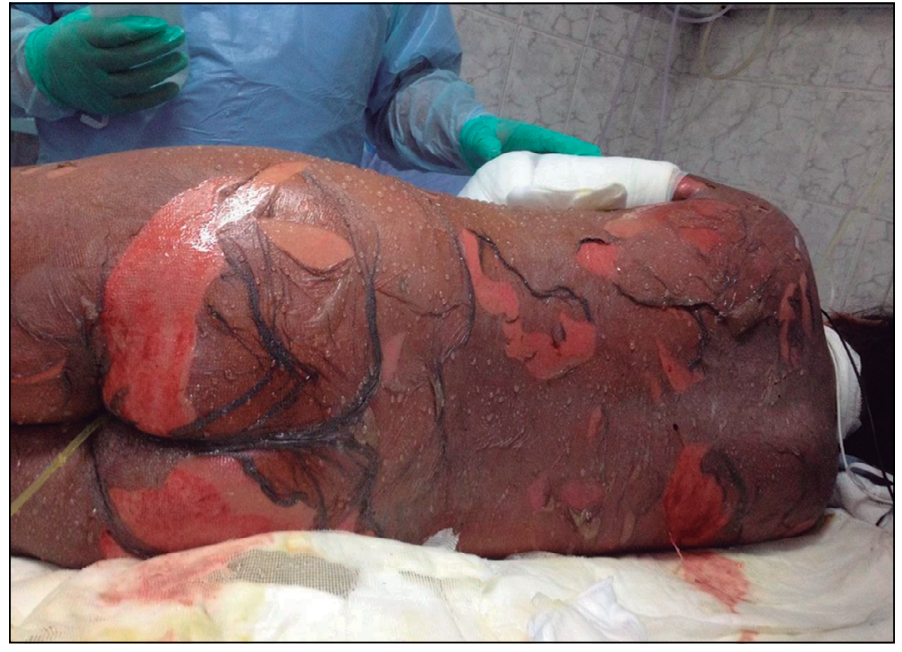

Figura 1. Extensa necrolisis epidermotóxica en la paciente.

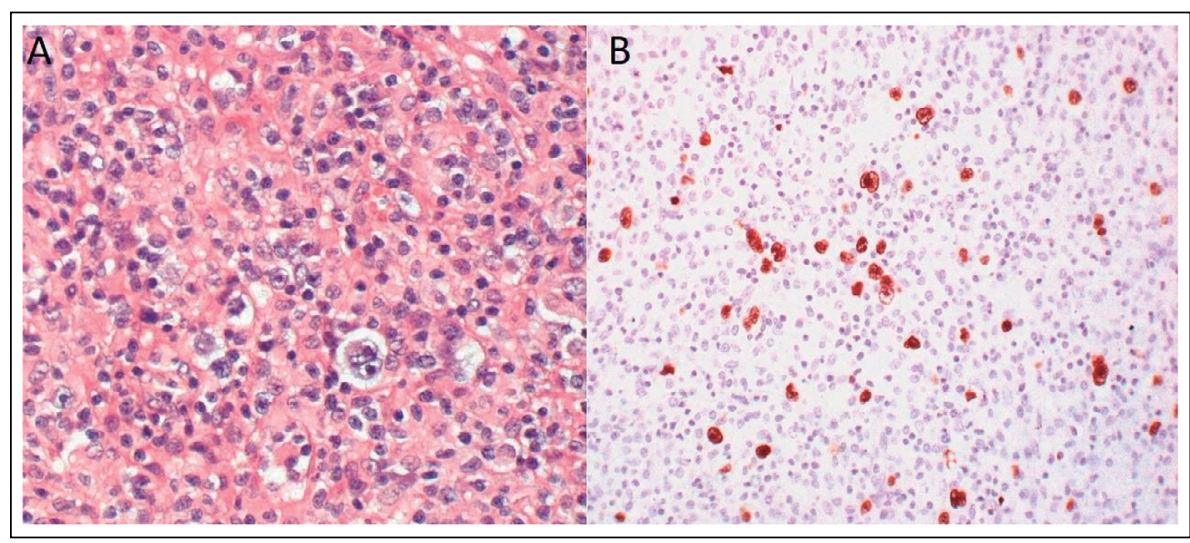

Figura 2. Biopsia de ganglio de la paciente. A. HE mostrando linfoma de Hodgkin. B. EBER por CISH positivo. 
CISH en la biopsia cutánea que resultó negativo. A las 2 semanas comenzó nuevamente con fiebre y pancitopenia, sin embargo dado el diagnóstico de LH se comenzó quimioterapia ABVD. Se realizaron 8 ciclos y actualmente se encuentra en remisión completa 3 años post quimioterapia. Examen físico, hemograma, TG, Fg y ferritina normales desde entonces.

\section{Discusión}

El SHF secundario es una entidad poco frecuente y letal. Fue descrito por primera vez en 19391. Se caracteriza por fiebre, pancitopenia, visceromegalias e inflamación descontrolada. En el SHF secundario o reactivo, las causas más frecuentes son las neoplásicas y las infecciosas, pero también se describe en enfermedades autoinmunes, secundario a drogas, quemaduras, entre otras. El SHF secundario a neoplasias es un diagnóstico desafiante, dado que su clínica y laboratorio se asemeja a clínica de linfoma, SIRS o falla multiorgánica por cualquier causa, lo que lleva a diagnóstico retrasado y mayor mortalidad. La incidencia mundial no se conoce con exactitud. En un estudio poblacional se estima la incidencia de SHF secundario a neoplasias en 0,36/100.000 habitantes/año. El SHF afectaría a 1\% de los adultos con neoplasias hematológicas, lo que podría aumentar hasta $20 \%$ en linfomas B o $\mathrm{T}^{2}$.

Dentro de las causas hematológicas, lo más frecuente son los linfomas no Hodgkin, tanto $\mathrm{T}$ como B. El LH da cuenta de sólo una minoría de los casos $(6 \%)^{3}$ y la mayoría son solo reportes de $\operatorname{casos}^{4-8}$. En un estudio de 34 pacientes con LH y SHF, se vio un promedio de edad de 46 años y todos fueron diagnosticados como etapa $\mathrm{IV}^{9}$, lo que concuerda con nuestro caso. Cabe mencionar que la mayoría de las neoplasias hematológicas con SHF están asociadas al virus Epstein Bar (VEB), como los linfomas T o NK 10-13. Los tipos de LH más frecuentemente asociado al SHF son el de celularidad mixta y depleción linfocítica, ambos con un alto porcentaje de VEB positivo, hasta $94 \%$ según Menard 9. En otros subtipos de LH esta asociación llega sólo hasta un $40 \%{ }^{14,15}$. Este caso se demostró mediante EBER la presencia del virus en las células neoplásicas. Es interesante también el hecho que dentro de las causas infecciosas, las más frecuentes son las virales, precisamente siendo el VEB el más publicado. Esto asociado a lo anteriormente mencionado sobre los linfomas asociados a VEB, abren la posibilidad que el VEB tenga un rol clave en la patogénesis del $\mathrm{SHF}^{9,16}$. El VEB infecta células B que estimulan Linfocitos T citotóxicos llevando a hipercitoquinemia, como IL2, INF alfa e IL6, que son los responsables finales del $\mathrm{SHF}^{17}$. Se describe además de lo anterior, que la proteína latente de membrana 1 (LMP-1) del VEB induce alta producción de TNFa por las células malignas infectadas, llevando a la hemofagocitosis e hipercitoquinemia descontrolada ${ }^{9,18}$. Esto nos lleva a la hipótesis que las células de Reed Stemberg infectadas (demostrado por EBER en nuestro caso) son las células que producen el incremento de citoquinas, llevando a SHF.

El manejo del SHF no está claro. Lo más ampliamente mencionado es el protocolo HLH0 ${ }^{15}$, pero este fue concebido en contexto de SHF primario (hereditario) en niños y como puente a trasplante de médula ósea, un escenario bastante diferente al nuestro. En nuestro caso la paciente remitió luego del uso de dexametasona e IgIV, que fue administrada por el cuadro de NET. La IgIV se ha usado con éxito en otros casos, especialmente en SHF secundarios asociados a virus. Es interesante que en este caso, el LH se asocia a VEB. La respuesta no fue duradera, pero nos dio el tiempo necesario para realizar biopsia y diagnóstico del linfoma. Posteriormente con la quimioterapia ABVD hizo remisión completa hasta ahora, tanto del linfoma como del SHF. La paciente sobrevivió a dos entidades letales con este manejo. Y lleva 3 años en remisión, lo que contrasta claramente el pronóstico descrito de SHF de sobrevida media de 2,1 meses en general y 1,4 meses en SHF asociado a neoplasias ${ }^{19}$. Existe alguna evidencia que la administración de IgIV más dexametasona es similar en respuesta al clásico protocolo HLH04 ${ }^{20}$.

Por otro lado, la NET es una muy infrecuente y potencialmente letal reacción cutánea aguda, generalmente asoaciado a drogas como sulfas, anticonvulsivantes, cefalosporinas y antiinflamatorios no esteroidales. En nuestro caso creemos que el Imipenem fue el gatillante. También puede verse secundario a virus, pricipalmente de la familia de los Herpes ${ }^{21,22}$. La incidencia estimada de 0,4 a 1,2/millón de habitantes/año. Es mediada por linfocitos CD8 activados que inducen apoptosis de keratinocitos. Tal como se describe, se manejó en 
una unidad de pacientes críticos y se administró IgIv, con lo que el cuadro cedió.

El SHF se asocia a manifestaciones cutáneas hasta en 65\% de los casos, siendo la mayoría erupciones cutáneas, rash, eritema maculopapular, eritroderma generalizado o púrpura. Sin embargo, existen muy pocos casos descritos de NET y SHF, y llama la atención la presencia de VEB en algunos de ellos ${ }^{23,24}$. Si bien es una muy infrecuente asociación, esta podría deberse a sobreposición de mecanismos fisiopatológicos, ya que en ambas entidades desarrollan una activación defectuosa de los LT citotóxicos CD8+ y elevación de granulosina sérica ${ }^{21,25}$. Incluso existen reportes de SHF secundario a VEB cuya necrosis epidérmica se atribuye directamente al virus y no a un NET como tall ${ }^{23}$. En ese caso se demostró infección de linfocitos T cutáneos con CISH para EBER, cosa que no pudimos demostrar en nuestro caso.

El pronóstico de ambas entidades es malo. La NET simula pacientes gran quemados, por lo que tienen gran riesgo de infecciones, falla multiorgánica y muerte. El SHF por su parte tiene una tasa de mortalidad aproximada de $40 \%$, y en SHF secundario a neoplasia la sobrevida global a 3 años es entre $18-55 \%$, siendo peor en los linfomas $\mathrm{T} /$ $\mathrm{NK}^{3}$. Por esta razón que la paciente lleve 3 años en remisión llama mucho la atención. Creemos que la remisión completa se debe al rápido reconocimiento y manejo de ambas patologías.

Según nuestro conocimiento este es el primer caso de NET asociado a un SHF secundario a LH, cuadro inusual, de presentación simultánea y con excelente evolución.

\section{Referencias}

1. Scott RB, Robb-Smith AH. Histiocytic medullary reticulocytosis. Lancet 1939; 2: 194-98.

2. Machaczka M, Vaktnäs J, Klimkowska M, Hägglund $\mathrm{H}$, Malignancy-associated hemophagocytic lymphohistiocytosis in adults: a retrospective population-based analysis from a single center. Leuk Lymphoma 2011; 52 (4): 613-9.

3. Ramos-Casals M, Brito-Zerón P, López-Guillermo A, Khamashta MA, Bosch X, Adult haemophagocytic síndrome, Lancet 2014; 383 (9927): 1503-16.

4. Kojima H, Takei N, Mukai Y, Hasegawa Y, Suzukawa $\mathrm{K}$, Nagata $\mathrm{M}$, et al. Hemophagocytic syndrome as the primary clinical symptom of Hodgkin's disease. Ann Hematol 2003; 82 (1): 53-6.
5. Preciado MV, De Matteo E, Fallo A, Chabay P, Drelichman G, Grinstein S. EBV-associated Hodgkin's disease in an HIV-infected child presenting with a hemophagocytic syndrome. Leuk Lymphoma 2001; 42: 231-4.

6. Dawson L, den Ottolander GJ, Kluin PM, Leeksma O. Reactive hemophagocytic syndrome as a presenting feature of Hodgkin's disease. Ann Hematol 2000; 79: 322-6.

7. Chim CS, Hui PK. Reactive hemophagocytic syndrome and Hodgkin's disease. Am J Hematol 1997; 55: 49-50.

8. Hasselblom S, Linde A, Riddel B. Hodgkin's lymphoma, Epstein-Barr virus reactivation and fatal hemophagocytic syndrome. J Intern Med 2004; 255: 289-95.

9. Ménard F, Besson C, Rincé P, Lambotte O, Lazure T, Canioni D, et al Hodgkin lymphomaassociated hemophagocytic syndrome: a disorder strongly correlated with Epstein-Barr virus, Clin Infect Dis 2008; 47 (4): 531-4.

10. Cheng AL, Su IJ, Chen YC, Uen WC, Wang CH, Characteristic clinicopathologic features of Epstein-Barr virus-associated peripheral T-cell lymphoma, Cancer. 1993; 72 (3): 909-16.

11. Gaffey M, Frierson H, Medeiros L, Weiss L, The relationship of Epstein-Barr virus to infection-related (sporadic) and familial hemophagocytic syndrome and secondary (lymphoma related) hemophagocytosis: an in situ hybridization study. Hum Pathol 1993; 24 (6): 657-67.

12. Iwatsuki K, Ohtsuka M, Harada H, Han G, Kaneko F. Clinicopathologic manifestations of Epstein-Barr virus associated cutaneous lymphoproliferative disorders. Arch Dermatol 1997; 133 (9): 1081-6.

13. Su IJ, Wang CH, Cheng AL, Chen RL, Hemophagocytic syndrome in Epstein-Barr virus associated T-lymphoproliferative disorders: disease spectrum, pathogenesis, and management. Leuk Lymphoma 1995; 19 (5-6): 401-6.

14. Okano M, Gross TG, Acute or Chronic Life-Threatening Diseases Associated With Epstein-Barr Virus Infection, Am J Med Sci 2012; 343 (6): 483-9.

15. Henter JI, Horne A, Aricó M, Egeler RM, Filipovich AH, Imashuku S, et al, HLH-2004: diagnostic and therapeutic guidelines for hemophagocytic lymphohistiocytosis. Pediatr Blood Cancer 2007; 48 (2): 124-31.

16. Jarrett RF, Stark GL, White J, Angus B, Alexander FE, Krajewski AS, et al. Impact of tumor Epstein-Barr virus status on presenting features and outcome in age-defined subgroups of patients with classic Hodgkin lymphoma: ampopulation-based study. Blood 2005; 106 (7): 2444-51.

17. Goudarzipour K, Kajiyazdi M, Mahdaviyani A, Eps- 
tein-Barr Virus-Induced Hemophagocytic Lymphohistiocytosis, Int J Hematol Oncol Stem Cell Res 2013; 7 (1): 42-5.

18. Janka GE. Familial and acquired hemophagocytic lymphohistiocytosis. Eur J Pediatr 2007; 166: 95-109.

19. Parikh SA, Kapoor P, Letendre L, Kumar S, Wolanskyj AP. Prognostic Factors and Outcomes of Adults With Hemophagocytic Lymphohistiocytosis . Mayo Clin Proc 2014; 89: 484-92.

20. Rajajee S, Ashok I, Manwani N, Rajkumar J, Gowrishankar K, Subbiah E. Profile of Hemophagocytic Lymphohistiocytosis; Efficacy of Intravenous Immunoglobulin Therapy. Indian J Pediatr 2014; 81 (12): 1337-41.

21. Lee HY, Chung WH. Toxic epidermal necrolysis: the year in review. Curr Opin Allergy Clin Immunol 2013; 13 (4): 330-6.

22. Schwartz RA, McDonough PH. Toxic epidermal ne- crolysis. Part I. Introduction, history, classification, clinical features, systemic manifestations, etiology and immunopathogenesis. J Am Acad Dermatol 2013; 69 (2): 173 .

23. Kawachi Y, Itoh M, Fujisawa Y, Furuta J, Nakamura Y, Banno T, et al. Epidermal cell necrosis with direct epidermal infiltration of Epstein-Barr virus (EBV)-encoded small nuclear RNA-positive T lymphocytes in a patient with EBV-associated haemophagocytic syndrome. Br J Dermatol 2007; 157 (5): 1053-6.

24. Sharma N, Clark J, Pham H, Efron D, MacGregor D, O'Keefe R, et al. TEN-like eruption in setting of EBV positive T-cell lymphoproliferative disease with HLH, in a child. Australas J Dermatol 2014; 55 (3): e44-7.

25. Nagasawa M, Ogawa K, Imashuku S, Mizutani S. Serum granulysin is elevated in patients with hemophagocytic lymphohistiocytosis. Int J Hematol 2007; 86 (5): 470-3. 